\title{
Research Article \\ Vectorial Form of Ekeland-Type Variational Principle in Locally Convex Spaces and Its Applications
}

\author{
S. Eshghinezhad and M. Fakhar \\ Department of Mathematics, Faculty of Sciences, University of Isfahan, Isfahan 81745-163, Iran \\ Correspondence should be addressed to M. Fakhar, majid_fakhar2000@yahoo.com
}

Received 23 June 2010; Accepted 2 November 2010

Academic Editor: A. T M. Lau

Copyright (C) 2010 S. Eshghinezhad and M. Fakhar. This is an open access article distributed under the Creative Commons Attribution License, which permits unrestricted use, distribution, and reproduction in any medium, provided the original work is properly cited.

By using a Daneš' drop theorem in locally convex spaces we obtain a vectorial form of Ekelandtype variational principle in locally convex spaces. From this theorem, we derive some versions of vectorial Caristi-Kirk's fixed-point theorem, Takahashi's nonconvex minimization theorem, and Oettli-Thera's theorem. Furthermore, we show that these results are equivalent to each other. Also, the existence of solution of vector equilibrium problem is given.

\section{Introduction and Preliminaries}

A very important result in nonlinear analysis about the existence result for an approximate minimizer of a lower semicontinuous and bounded below function was first presented by Ekeland [1]. Known nowadays as Ekeland's variational principle (in short, EVP), it has significant applications in the geometry theory of Banach spaces, optimization theory, game theory, optimal control theory, dynamical systems, and so forth; see [1-11] and references therein. It is well known that EVP is equivalent to many famous results, namely, the CaristiKirk fixed-point theorem, the petal theorem, Phelp's lemma, Danês' drop theorem, OettliThéra's theorem and Takahashi's theorem, see, for example, [4, 6, 7, 10, 12-19]. Many authors have obtained EVP on complete metric spaces $[1,10,19,20]$ and in locally convex spaces [20-24]. Along with the development of vector optimization and motivated by the wide usefulness of EVP, many authors have been interested in obtaining this principle for vector-valued functions and set-valued mappings; see [3, 5, 8, 9, 11-15, 21, 22, 25]. Recently, this principle has been obtained for bifunctions and applied to solve equilibrium problem in nonconvex setting [10,12, 14-16, 20, 26, 27]. Our goal in this paper is to obtain Ekeland's variational principle for vector-valued bifunctions in locally convex spaces. By 
using this result we derive the existence of solution of vector equilibrium problem in the setting of seminormed spaces. Also, we obtain vectorial Caristi-Kirk's fixed-point theorem, vectorial Takahashi's nonconvex minimization theorem and vectorial Oettli-Théra's theorem. Moreover, we show that these results and Daneš' drop theorem are equivalent to each other. Let us, introduce some known definitions and results which will be used in the sequel.

Let $E$ be a Hausdorff locally convex real vector space. A subset $B$ of $E$ is said to be a disc, if $B$ is bounded and absolutely convex. Let $\mathrm{sp}[B]$ be the vector subspace spanned by $B$, and $p_{B}$ be the Minkowski functional of $B$, then $E_{B}:=\left(\operatorname{sp}[B], p_{B}\right)$ is a normed space. If $E_{B}$ is a Banach space, then $B$ is called a Banach disc. A sequence $\left\{x_{n}\right\}$ in $E$ is said to be locally convergent to an element $x$ if there is a disc $B$ in $E$ such that the sequence $\left\{x_{n}\right\}$ is convergent to $x$ in $E_{B}$ and $\left\{x_{n}\right\}$ is said to be locally Cauchy if there is a disc $B$ in $E$ such that $\left\{x_{n}\right\}$ is a Cauchy sequence in $E_{B}$. We say that $E$ is a locally complete space if every locally Cauchy sequence is locally convergent. This is equivalent to that each bounded subset of $E$ is contained in a certain Banach disc. A nonempty subset $X$ of $E$ is said to be locally complete if every locally Cauchy sequence in $X$ is locally convergent to a point in $X$. The subset $X$ is said to be locally closed if for any locally convergent sequence in $X$, its local limit point belongs to $X$. It is well known that every sequentially complete locally convex space is locally complete and the converse is not true; see [28, 29].

Let $(Y, C)$ be a locally convex space ordered by the nontrivial closed convex cone $C$ as follows:

$$
x \leq_{C} y \Longleftrightarrow y-x \in C .
$$

For every $x, y \in Y$ we write

$$
x \mathbb{L}_{C} y \Longleftrightarrow x-y \notin-C .
$$

Definition 1.1. Let $X$ be a nonempty subset of a locally convex space $E,(Y, C)$ be a locally convex space ordered by the nontrivial closed convex cone $C$. A vector-valued function $\phi$ : $X \rightarrow Y$ is said to be

(1) $(e, C)$-locally lower semicontinuous if for every $r \in \mathbb{R}$ the set $\left\{x \in X: \phi(x) \leq_{C} r e\right\}$ is locally closed in $X$;

(2) $C$-upper semicontinuous at $x_{0} \in X$ if for any neighborhood $U$ of $\phi\left(x_{0}\right)$, there exists a neighborhood $V$ of $x_{0}$ such that $\phi(x) \in U-C$, for all $x \in V$. If $\phi$ is $C$-upper semicontinuous at each point of $X$, then $\phi$ is said to be $C$-upper semicontinuous on $X$;

(3) C-bounded from below, if there exists $b \in Y$ such that $b \leq_{C} \phi(x)$ for all $x \in X$.

Assume that the interior of $C$ (int $C$ ) is nonempty and $g: X \times X \rightarrow Y$ is a vector-valued function. The vector equilibrium problem (in short, VEP) is to find $\bar{x} \in \mathrm{X}$ such that

$$
g(\bar{x}, y) \notin-\operatorname{int} C \quad \forall y \in X .
$$

It is well known that VEP includes fundamental mathematical problems like vector optimization, vector variational inequality, and vector complementarity problem. For further details on VEP, one can refer to [23, 24, 30-32]. 
Let $e \in \operatorname{int} C$. Recall the definition of the Gerstewitz function [33]:

$$
\xi_{e}(y):=\inf \{r \in \mathbb{R}: y \in r e-C\}, \quad \forall y \in Y .
$$

The following lemma describes some properties of the Gerstewitz function and it will be used in the sequel. For its proof we refer the reader to $[5,6,33]$.

Lemma 1.2. For each $r \in \mathbb{R}$ and $y \in Y$, the following statements are satisfied:

(i) $\xi_{e}(y) \leq r \Leftrightarrow y \leq_{C} r e$.

(ii) $\xi_{e}(y)>r \Leftrightarrow y \mathbb{L}_{C} r e$.

(iii) $\xi_{e}(y) \geq r \Leftrightarrow y \notin r e-\operatorname{int} C$.

(iv) $\xi_{e}(y)<r \Leftrightarrow y \in r e-\operatorname{int} C$.

(v) $\xi_{e}(\cdot)$ is positively homogeneous and continuous on $Y$.

(vi) $\xi_{e}\left(y_{1}+y_{2}\right) \leq \xi_{e}\left(y_{1}\right)+\xi_{e}\left(y_{2}\right)$, for all $y_{1}, y_{2} \in Y$.

(vii) $\xi_{e}(\cdot)$ is monotone, that is, if $y_{2} \in y_{1}+C$, then $\xi_{e}\left(y_{1}\right) \leq \xi_{e}\left(y_{2}\right)$.

In order to obtain a vectorial form of Ekeland-type variational principle we need the following result.

Theorem 1.3 (see [17]). Let $A$ be a locally closed subset of a locally convex space $E$ and $B$ a locally closed, bounded convex subset of $E$ with $0 \notin \mathrm{cl}(A-B)$. If either $A$ or $B$ is locally complete, then for each $x_{0} \in A$, there exists $a \in D\left(x_{0}, B\right) \cap A$ such that $D(a, B) \cap A=\{a\}$, where $D(a, B)$ denotes the convex hull of $\{a\} \cup B$.

\section{Vectorial Ekeland-Type Variational Principle}

Recently, Qiu [18] obtained some versions of Ekeland's variational principle in locally convex spaces, which only need to assume local completeness of some related sets. Motivated by this paper we obtain some versions of EVP for vector-valued bifunctions in locally convex spaces. These results extend Qiu's results to vector-valued bifunctions.

Throughout this section $E$ is a locally convex space, $X$ is locally closed subset of $E,\left\{p_{\lambda}\right\}_{\lambda \in \Lambda}$ is a family of seminorms generating the locally convex topology on $E, Y$ is a Hausdorff locally convex space ordered by a closed convex cone $C$ with int $C \neq \emptyset$ and $e \in \operatorname{int} C$. We consider a vector-valued bifunction $f: X \times X \rightarrow Y$, a family of positive real numbers $\left\{\alpha_{\lambda}\right\}_{\lambda \in \Lambda}$ and the following assumptions:

(A1) $f(x, x)=0$ for all $x \in X$.

(A2) $f(z, x) \leq_{C} f(z, y)+f(y, x)$ for any $x, y, z \in X$.

(A3) $y \rightarrow f(x, y)$ is C-bounded from below for all $x \in X$.

(A4) $y \rightarrow f(x, y)$ is $(e, C)$-locally lower semicontinuous for any $x \in X$.

(A5) There exists $x_{0} \in X$ such that the set $\left\{x \in X: f\left(x_{0}, x\right) \leq_{C} 0\right\}$ is locally complete.

(A6) The set $\bigcap_{\lambda \in \Lambda}\left\{x \in E: \alpha_{\curlywedge} p_{\lambda}(x) \leq 1\right\}$ is locally complete.

Notice that if assumptions (A1) and (A2) hold, then $f$ is called half distance. The following result is a vectorial form of Ekeland-type variational principle. 
Theorem 2.1. Suppose that assumptions (A1)-(A4) are satisfied. If either assumption (A5) or assumption (A6) holds, then for any $\varepsilon>0$, there exists $z \in X$ such that

(i) $f\left(x_{0}, z\right)+\varepsilon \alpha_{\lambda} p_{\lambda}\left(z-x_{0}\right) e \leq_{C} 0$, for any $\lambda \in \Lambda$;

(ii) For any $x \neq z$, there exists $\mu \in \Lambda$ such that $f(z, x)+\varepsilon \alpha_{\mu} p \mu(x-z) e \mathbb{L}_{C} 0$.

Proof. Without loss of generality, we may assume that $\varepsilon=1$ and put $M:=E \times \mathbb{R}$ with the product topology, then the topology can be generated by a family $\left\{q_{\lambda}: \lambda \in \Lambda\right\}$ of seminorms, where $q_{\lambda}(x, t)=p_{\lambda}(x)+|t|$, for all $(x, t) \in M$. If $A=\left\{(x, t) \in X \times \mathbb{R}: f\left(x_{0}, x\right) \leq_{C} t e, t \leq 0\right\}$ and $m:=\inf \{t:(x, t) \in A\}$, then since $y \rightarrow f(x, y)$ is $C$-bounded from below for all $x \in X$ we have $-\infty<m \leq 0$. Take any fixed real number $r<m$ and put $B:=\left\{(x, r) \in M: \alpha_{\lambda} p_{\lambda}\left(x-x_{0}\right) \leq\right.$ $-r, \forall \lambda \in \Lambda\}$. Then $K:=\operatorname{cone}(B)$ is exactly the set $\left\{(y, t) \in M: \alpha_{\lambda} p_{\lambda}\left(y-x_{0}\right) \leq-t\right.$, for all $\lambda \in \Lambda\}$. If the set $\left\{x \in X: f\left(x_{0}, x\right) \leq_{C} 0\right\}$ is locally complete, then $A$ is locally complete and if $\bigcap_{\lambda \in \Lambda}\left\{x \in E: \alpha_{\lambda} p_{\lambda}(x) \leq 1\right\}$ is locally complete, then $B$ is locally complete. Furthermore, $B$ is bounded closed convex subset of $M$ and $q_{\lambda}(A-B) \geq m-r>0$. Hence, by Theorem 1.3, there exists

$$
(z, s) \in A \cap D((0,0), B) \subset A \cap K
$$

such that

$$
A \cap D((z, s), B)=\{(z, s)\} .
$$

According to (2.1), we have $(z, s) \in A \cap K$, so

$$
f\left(x_{0}, z\right) \leq_{C} e s
$$

and for each $\lambda \in \Lambda$

$$
\alpha_{\curlywedge} p_{\lambda}\left(z-x_{0}\right) e \leq_{C}-e s
$$

Therefore, by (2.3) and (2.4), we have

$$
\alpha_{\lambda} p_{\lambda}\left(z-x_{0}\right) e \leq_{C}-e s \leq_{C}-f\left(x_{0}, z\right), \quad \forall \lambda \in \Lambda .
$$

Hence, the part (i) holds. We show that the point $z$ satisfies in the part (ii). Let $\delta=$ $\xi_{e}\left(f\left(x_{0}, z\right)\right)-r / s-r$. Since $r e \leq_{C} m e \leq_{C} f\left(x_{0}, z\right) \leq_{C}$ se and $\xi_{e}$ is monotone, then $0 \leq \delta \leq 1$. On the other hand we have $\delta s+(1-\delta) r=\xi_{e}\left(f\left(x_{0}, z\right)\right)$. Hence,

$$
\left(z, \xi_{e}\left(f\left(x_{0}, z\right)\right)\right)=(z, \delta s+(1-\delta) r)=\delta(z, s)+(1-\delta)(z, r)
$$

But from (2.5) we have

$$
\alpha_{\lambda} p_{\lambda}\left(z-x_{0}\right) e \leq_{C}-f\left(x_{0}, z\right) \leq_{C}-r e, \quad \forall \lambda \in \Lambda .
$$


Therefore, $(z, r) \in B$. Thus, $\left(z, \xi_{e}\left(f\left(x_{0}, z\right)\right)\right) \in D((z, s), B)$. Also, clearly $\left(z, \xi_{e}\left(f\left(x_{0}, z\right)\right)\right) \in A$. Hence, we have

$$
\left(z, \xi_{e}\left(f\left(x_{0}, z\right)\right)\right) \in A \cap D((z, s), B)
$$

Therefore, by $(2.1),\left(z, \xi_{e}\left(f\left(x_{0}, z\right)\right)\right)=\{(z, s)\}$ and so $s=\xi_{e}\left(f\left(x_{0}, z\right)\right)$.

Supposing that $x \in X$ and $x \neq z$, we consider the following two cases.

Case 1. If $\left(x, \xi_{e}\left(f\left(x_{0}, x\right)\right)\right) \notin A$, then $\xi_{e}\left(f\left(x_{0}, x\right)\right)>0$. Since $\xi_{e}$ is monotone, then for all $\lambda \in \Lambda$ we have

$$
\xi_{e}\left(f\left(x_{0}, x\right)+\alpha_{\lambda} p_{\lambda}(z-x)\right) e \geq \xi_{e}\left(f\left(x_{0}, x\right)\right)>0 \geq \xi_{e}\left(f\left(x_{0}, z\right)\right) .
$$

But $f$ is half distance and $\xi_{e}$ is sublinear, thus

$$
\begin{aligned}
\xi_{e}\left(f(z, x)+\alpha_{\curlywedge} p_{\lambda}(z-x) e\right) & \geq \xi_{e}\left(f\left(x_{0}, x\right)-f\left(x_{0}, z\right)+\alpha_{\curlywedge} p_{\lambda}(z-x) e\right) \\
& \geq \xi_{e}\left(\left(f\left(x_{0}, x\right)+\alpha_{\curlywedge} p_{\lambda}(z-x) e\right)-\xi_{e} f\left(x_{0}, z\right)\right)>0 .
\end{aligned}
$$

Hence, by the part (ii) of Lemma 1.2;

$$
f(z, x)+\alpha_{\curlywedge} p_{\lambda}(z-x) e \not_{C} 0 .
$$

Case 2. Let $\left(x, \xi_{e}\left(f\left(x_{0}, x\right)\right)\right) \in A$, we will show that $\left(x, \xi_{e}\left(f\left(x_{0}, x\right)\right)\right) \notin(z, s)+K$. If not, we assume that $\left(x-z, \xi_{e}\left(f\left(x_{0}, x\right)\right)-s\right) \in K$, that is,

$$
s-\xi_{e}\left(f\left(x_{0}, x\right)\right) \geq \alpha_{\curlywedge} p_{\lambda}(x-z), \quad \forall \lambda \in \Lambda
$$

Since $x \neq z$ and $\left\{p_{\lambda}\right\}_{\lambda \in \Lambda}$ separates points in $X$, we conclude that there exists $\mu \in \Lambda$ such that $p_{\mu}(x-z)>0$ thus $s-\xi_{e}\left(f\left(x_{0}, x\right)\right)>0$. Put

$$
\eta=\frac{s-\xi_{e}\left(f\left(x_{0}, x\right)\right)}{s-r}, \quad 0<\eta<1 .
$$

Since $K$ is a cone,

$$
\left(\frac{x-z}{\eta}, \frac{\xi_{e}\left(f\left(x_{0}, x\right)\right)-s}{\eta}\right) \in K
$$

that is,

$$
\left(\frac{x-z}{\eta}, r-s\right) \in K
$$


By (2.1),

$$
(z, s) \in K
$$

Since $K$ is a convex cone, by (2.15) and (2.16) we have

$$
(z, s)+\left(\frac{x-z}{\eta}, r-s\right) \in K
$$

so

$$
\left(z+\frac{x-z}{\eta}, r\right) \in K \cap(E \times\{r\})=B .
$$

It is easy to verify that

$$
(1-\eta) s+\eta r=\frac{\xi_{e}\left(f\left(x_{0}, x\right)\right)-r}{s-r} s+\frac{s-\xi_{e}\left(f\left(x_{0}, x\right)\right)}{s-r}=\xi_{e}\left(f\left(x_{0}, x\right)\right) .
$$

Hence,

$$
\left(x, \xi_{e}\left(f\left(x_{0}, x\right)\right)\right) \in D((z, s), B) \cap A=\{(z, s)\}
$$

Therefore, $\left(x, \xi_{e}\left(f\left(x_{0}, x\right)\right)\right)=(z, s)$ and so $x=z$, which it is a contradiction. This shows that $\left(x, \xi_{e}\left(f\left(x_{0}, x\right)\right)\right) \notin(z, s)+K$. Thus, there exists $\mu \in \Lambda$ such that

$$
\xi_{e}\left(f\left(x_{0}, x\right)\right)-\xi_{e}\left(f\left(x_{0}, z\right)\right)+\alpha_{\mu} p_{\mu}(x-z)>0
$$

On the other hand by (A2) we have

$$
\xi_{e}(f(z, x)) \geq \xi_{e}\left(f\left(x_{0}, x\right)\right)-\xi_{e}\left(f\left(x_{0}, z\right)\right) .
$$

Hence,

$$
\xi_{e}\left(f(z, x)+\alpha_{\mu} p_{\mu}(x-z) e\right)>0
$$

Therefore,

$$
f(z, x)+\alpha_{\mu} p_{\mu}(x-z) e \underline{\leq}_{C} 0 .
$$


Remark 2.2. In the above theorem, if assumption (A5) holds, then instead of assumption (A3), we can assume that $y \mapsto f\left(x_{0}, y\right)$ is $C$-bounded from below. Also, if assumption (A6) holds, assumption (A3) can be replaced by the following assumption: $y \mapsto f(x, y)$ is $C$-bounded from below for some $x \in X$.

As a consequence of the above theorem we can obtain the following result which is a vectorial version of Theorem 3.1 of [18].

Corollary 2.3. Let $\phi: X \rightarrow Y$ be a function such that $\phi$ is $C$-bounded from below and $\phi$ is $(e, C)$ locally lower semicontinuous. Furthermore, let assumption (A6) holds or there exists $x_{0} \in X$ such that the set $\left\{x \in X: \phi(x) \leq_{C} \phi\left(x_{0}\right)\right\}$ is locally complete. Then there exists $z \in X$ such that

(i) $\phi(z)+\varepsilon \alpha_{\curlywedge} p_{\lambda}\left(z-x_{0}\right) e \leq_{C} \phi\left(x_{0}\right)$, for any $\lambda \in \Lambda$;

(ii) for any $x \neq z$, there exists $\mu \in \Lambda$ such that $\phi(x)+\varepsilon \alpha_{\mu} p \mu(x-z) e \mathbb{E}_{C} \phi(z)$.

Proof. It is enough in Theorem 2.1 to consider $f(x, y)=\phi(y)-\phi(x)$ for all $x, y \in X$. other.

In the following theorem we show that the previous results are equivalent to each

Theorem 2.4. Corollary 2.3 implies Theorem 2.1.

Proof. Let $\phi: X \rightarrow Y$ be defined as follows:

$$
\phi(x)=f\left(x_{0}, x\right) \quad \forall x \in X
$$

It is an easy task to derive the assumptions of Corollary 2.3 for the above function from the assumptions of Theorem 2.1. Therefore, there exists $z \in X$ which satisfies the conditions (i) and (ii) of Corollary 2.3. Hence,

(i) $f\left(x_{0}, z\right)+\varepsilon \alpha_{\lambda} p_{\lambda}\left(z-x_{0}\right) e \leq_{C} 0$, for any $\lambda \in \Lambda$;

(ii) for any $x \neq z$, there exists $\mu \in \Lambda$ such that $f\left(x_{0}, x\right)-f\left(x_{0}, z\right)+\varepsilon \alpha_{\mu} p \mu(x-z) e \not_{C} 0$.

Also, by assumption (A2) we have $f\left(x_{0}, x\right) \leq_{C} f\left(x_{0}, z\right)+f(z, x)$. Thus,

$$
\begin{aligned}
& f(z, x)+f\left(x_{0}, z\right)-f\left(x_{0}, x\right)+f\left(x_{0}, x\right)-f\left(x_{0}, z\right)+\varepsilon \alpha_{\mu} p \mu(x-z) e \\
& =f(z, x)+\varepsilon \alpha_{\mu} p \mu(x-z) e \mathbb{L}_{C} 0 .
\end{aligned}
$$

Let $S$ be a convex subset of $E$ containing 0 . The Minkowski functional of $S$ is defined as follows:

$$
p_{S}(x)= \begin{cases}\inf \{t>0: x \in t S\} & \text { if there exists } t>0 \text { such that } x \in t A \\ +\infty, & \text { otherwise. }\end{cases}
$$

We extend $Y$ by an additional element $\infty^{*}$ such that $\infty^{*}+y \mathbb{L}_{C} 0$ for all $y \in Y$ and $c \times(+\infty)=\infty$ * for all $c \in \operatorname{int} C$. 
By using Theorem 2.1 we obtain another version of vectorial form of Ekeland-type variational principle in which the perturbation function is the Minkowski functional of a bounded set.

Theorem 2.5. Suppose that assumptions (A1)-(A4) are satisfied. Let $S \subseteq E$ be a locally closed, bounded convex set containing 0 and $\alpha$ be a positive real number. Let $S$ be locally complete or assumption (A5) holds. Then, for any $\varepsilon>0$, there exists $z \in X$ such that:

(i) $f\left(x_{0}, z\right)+\varepsilon \alpha p_{S}\left(z-x_{0}\right) e \leq_{C} 0$;

(ii) For any $x \neq z, f(z, x)+\varepsilon \alpha p_{S}(x-z) e \overleftarrow{L}_{C} 0$.

Proof. Suppose that $T$ is the absolutely convex hull of the set $S \cup\left\{x_{0}\right\}$, then $\left(X_{T}, p_{T}\right)$ is a normed space. Assume that

$$
D:=\left\{x \in X_{T}: f\left(x_{0}, x\right)+\varepsilon \alpha p_{S}\left(x-x_{0}\right) e \leq_{C} 0\right\}
$$

Since $x_{0} \in T$, then $x_{0} \in D$. Also, $f\left(x_{0}, \cdot\right)$ is $(e, C)$-locally lower semicontinuous and $p_{S}$ is locally lower semicontinuous, then $D$ is closed in $\left(X_{T}, p_{T}\right)$. Suppose that $g$ is restricted $f$ to $D \times D$. If $S$ is locally complete then $T$ is a Banach disk and $\left(X_{T}, p_{T}\right)$ is a Banach space. If the set $\left\{x \in X: f\left(x_{0}, x\right) \leq_{C} 0\right\}$ is locally complete, then $\left.\left\{x \in D: g\left(x_{0}, x\right) \leq_{C} 0\right)\right\}=D \cap\{x \in X:$ $\left.f\left(x_{0}, x\right) \leq_{C} 0\right\}$ is a complete set in $\left(X_{T}, p_{T}\right)$. Therefore, by Theorem 2.1 there exists $z \in D$ such that:

(a) $g\left(x_{0}, x\right)+\varepsilon \alpha p_{T}\left(z-x_{0}\right) e \leq_{C} 0$.

(b) For any $x \in D$ and $x \neq z$,

$$
g(z, x)+\varepsilon \alpha p_{T}(x-z) e \not_{C} 0 .
$$

Since $z \in D$, then the part (a) holds. Now, we show that the part (b) holds. If $x \neq z$ and $x \in D$, then (2.29) becomes

$$
f(z, x)+\varepsilon \alpha p_{S}(x-z) e \not_{C} 0 .
$$

Let $x \neq z$ and $x \notin X_{T}$, then $p_{S}(x-z)=+\infty$, so the part (b) holds. Let $x \neq z, x \in X_{T}, x \in X \backslash D$ and $f(z, x)+\varepsilon \alpha p_{S}(z-x) e \leq_{C} 0$. Since $f\left(x_{0}, z\right)+\varepsilon \alpha p_{S}\left(z-x_{0}\right) e \leq_{C} 0$, then

$$
f\left(x_{0}, x\right)+\operatorname{\varepsilon \alpha p}_{S}\left(x-x_{0}\right) e \leq_{C} f\left(x_{0}, z\right)+\varepsilon \alpha p_{S}\left(z-x_{0}\right) e+f(z, x)+\varepsilon \alpha p_{S}(z-x) e \leq_{C} 0 .
$$

Therefore, $x \in D$ which is a contradiction. Hence,

$$
f(z, x)+\varepsilon \alpha p_{S}(z-x) e \not_{C} 0, \quad \forall x \in X \backslash D .
$$


Assuming that $E$ is a locally complete locally convex space, the condition on local completeness of some related subsets is automatically satisfied. However, we give the following examples of spaces which are not locally complete but the condition on local completeness of some related subsets is satisfied.

Example 2.6. Let $C[0,1]$ be the space of all continuous functions defined on $[0,1]$. By Corollary $11-7-3,11-7-4$ of $[29], C[0,1]$ with weak-topology is quasi barreled but it is not barreled. Therefore, by Proposition 11-2-5 of [29], M[0,1] with weak*-topology is not locally complete. Moreover,

$$
\bigcap_{x \in C[0,1]}\left\{x^{*} \in M[0,1]:\left|\left\langle x^{*}, x\right\rangle\right| \leq 1\right\}=\left\{x^{*} \in M[0,1]:\left\|x^{*}\right\| \leq 1\right\} .
$$

But by Banach-Alaoglu theorem this set is weak ${ }^{*}$-compact. Also $C[0,1]$ is separable, so weak*topology on unit ball $M[0,1]$ is metrizable. Hence, this set is locally complete.

Example 2.7. Let $\left(C^{1}[0,1],\|\|_{\infty}\right)$ be the space of all differentiable functions whose derivative is continuous. Then $\left(C^{1}[0,1],\|\|_{\infty}\right)$ is not a complete space. Therefore, by Proposition 5.1 .9 [28] $\left(C^{1}[0,1],\|\|_{\infty}\right)$ is not a locally complete space.

Also, the set $\left\{x \in C^{1}[0,1]:\|x\|_{\infty} \leq 1\right\}$ is not locally complete. Suppose that $f: C^{1}[0,1] \times C^{1}[0,1] \rightarrow C[0,1]$ is defined as follows:

$$
f(x, y)=y^{2}-x^{2} \quad \forall x, y \in C^{1}[0,1],
$$

where $C[0,1]$ is ordered by the cone $C=\{x \in C[0,1]: x(t) \geq 0\}$. If we choose $x_{0}=0$, then the set $\left\{x \in C^{1}[0,1]: x^{2}(t) \leq x_{0}^{2}(t)=0, \forall t \in[0,1]\right\}=\{0\}$ is locally complete.

\section{Caristi-kirk's Fixed-Point Theorem, Takahashi's Nonconvex Minimization Theorem, and Oettli-Théra's Theorem and Equilibrium Problem}

In this section, we obtain an existence result for solution of vector equilibrium problem in nonconvex setting. Also, some new versions of the vectorial Caristi-Kirk fixed-point theorem, vectorial Takahashi's nonconvex minimization theorem and the vectorial OettliThéra theorem are given.

Theorem 3.1. Let $X$ be a weakly compact subset of a semi normed space $(Z, p)$. Suppose that $f$ : $X \times X \rightarrow Y$ is a function satisfying assumptions (A1)-(A5) together with some $x_{0} \in X$ and $f(\cdot, y)$ is $C$-upper semicontinuous for every $y \in X$. Then, there exists $\bar{x} \in X$ such that

$$
f(\bar{x}, y) \notin-\operatorname{int} C, \quad \forall y \in X .
$$

Proof. Assume that $S=\{x \in Z: p(x) \leq 1\}$, then $p_{S}(x)=p(x)$ for all $x \in Z$. Taking $\varepsilon=1 / n$ and $\alpha=1$, from Theorem 2.5, we find a sequence $\left\{x_{n}\right\}$, such that

$$
f\left(x_{n}, y\right)+\frac{1}{n} p\left(x_{n}-y\right) e \underline{\Sigma}_{C} 0, \quad \forall y \neq x_{n}
$$


By the weakly compactness of $X$, we can assume that $\left\{x_{n}\right\}$ weakly converges to $\bar{x} \in X$. Suppose that $f(\bar{x}, \bar{y}) \in-\operatorname{int} C$ for a suitable $\bar{y} \in X$. Take a neighborhood $U$ of $f(\bar{x}, \bar{y})$ such that $U \subset-\operatorname{int} C$. By $C$-upper semicontinuity of $f(\cdot, \bar{y})$, there exists a natural number $N$ such that $f\left(x_{n}, \bar{y}\right) \in U-C$, for $n \geq N$. Moreover, if $n$ is big enough, $(1 / n) p\left(x_{n}-\bar{y}\right) e+U \subseteq-\operatorname{int} C$, thus

$$
f\left(x_{n}, \bar{y}\right)+\frac{1}{n} p\left(x_{n}-\bar{y}\right) e \in U-C+\frac{1}{n} p\left(x_{n}-\bar{y}\right) e \subseteq-\operatorname{int} C
$$

This is a contradiction.

In the above theorem, when $X$ is not necessarily weakly compact we have the following result. Since its proof is similar to Theorem 4 of [20], we omit it.

Theorem 3.2. Let $X$ be a nonempty subset of a reflexive semi normed space $(Z, p)$. Suppose that $f: X \times X \rightarrow Y$ is a function satisfying assumptions (A1)-(A5) together with some $x_{0} \in X$ and $f(\cdot, y)$ is C-upper semicontinuous for every $y \in X$. Let the following coercivity condition holds:

There exists a nonempty closed bounded subset $K$ of $X$ such that for all $x \in X \backslash K$ there exists $y \in X$ with $p(y)<p(x)$ satisfying $f(x, y) \leq_{C} 0$.

Then, there exists $\bar{x} \in X$ such that

$$
f(\bar{x}, y) \notin-\operatorname{int} C, \quad \forall y \in X
$$

As a consequence of Theorems 2.1 and 2.5 we can obtain two versions of vectorial Caristi-Kirk's fixed-point theorem.

Theorem 3.3. Suppose that all of the conditions of Theorem 2.1 are satisfied. Assume that $T: X \rightarrow$ $2^{X}$ is a set-valued mapping with nonempty valued and the following property holds:

$$
f(x, y)+\alpha_{\lambda} p_{\lambda}(y-x) e \leq_{C} 0 ; \quad \forall \lambda \in \Lambda, \forall x \in X, \forall y \in T(x)
$$

Then, there exists $z \in T\left(x_{0}\right)$ such that $T z=\{z\}$.

Proof. Let $z$ be a point which satisfies in the parts (i) and (ii) with $\varepsilon=1$. We show that $T z=\{z\}$. If there exists $x \in T z$ and $x \neq z$, then by Theorem 2.1 there is a $\mu \in \Lambda$ such that $f(z, x)+\alpha_{\mu} p_{\mu}(x-z) e \mathbb{L}_{C} 0$ and it is a contradiction.

The proof of the following results is similar to that of Theorem 3.3 and we omit it.

Theorem 3.4. Suppose that all of the conditions of Theorem 2.5 are satisfied and $T: X \rightarrow 2^{X}$ is a set-valued mapping with nonempty valued and the following condition holds:

$$
f(x, y)+\alpha p_{S}(y-x) e \leq_{C} 0 ; \quad \forall x \in X, y \in T(x) .
$$

Then, there exists $z \in T\left(x_{0}\right)$ such that $T z=\{z\}$.

In the following we give two versions of vectorial Takahashi's nonconvex minimization. 
Theorem 3.5. Suppose that all of conditions of Theorem 2.1 are satisfied and for each $y \in X$ with $f\left(x_{0}, X\right) \cap\left(f\left(x_{0}, y\right)-C\right) \neq\left\{f\left(x_{0}, y\right)\right\}$, there exists $x \neq y$ such that $f(y, x)+e \alpha_{\lambda} p_{\lambda}(x-y) \leq_{C} 0$ for any $\lambda \in \Lambda$. Then, there exists $\bar{x} \in X$ such that $f\left(x_{0}, \bar{x}\right) \in \operatorname{Min} f\left(x_{0}, X\right)$ that is $f\left(x_{0}, X\right) \cap\left(f\left(x_{0}, \bar{x}\right)-C\right)=$ $\left\{f\left(x_{0}, \bar{x}\right)\right\}$.

Proof. By Theorem 2.1 there exists $z \in X$ such that for each $x \neq z$ there exists $\mu \in \Lambda$ such that $f(z, x)+e \alpha_{\mu} p_{\mu}(x-z) \mathbb{Z}_{C} 0$. If $f\left(x_{0}, X\right) \cap\left(f\left(x_{0}, z\right)-C\right) \neq\left\{f\left(x_{0}, z\right)\right\}$, then by assumption there exists $x \neq z$ such that $f(z, x)+e \alpha_{\mu} p_{\mu}(x-z) \leq_{C} 0$, and this is a contradiction.

The proof of the following results is similar to that of Theorem 3.5 and we omit it.

Theorem 3.6. Suppose that all of the conditions of Theorem 2.5 are satisfied and for each $y \in X$ with $f\left(x_{0}, X\right) \cap\left(f\left(x_{0}, y\right)-C\right) \neq\left\{f\left(x_{0}, y\right)\right\}$, there exists $x \neq y$ such that $f(y, x)+e p_{S}(x-y) \leq_{C} 0$. Then, there exists $\bar{x} \in X$ such that $f\left(x_{0}, \bar{x}\right) \in \operatorname{Min} f\left(x_{0}, X\right)$.

In the final step we obtain a vectorial form of Oettli-Théra type theorem.

Theorem 3.7. Assume that all of the conditions of Theorem 2.1 are satisfied and $S_{0}=\{x \in X$ : $\left.f\left(x_{0}, x\right)+\varepsilon \alpha_{\lambda} p_{\lambda}\left(x-x_{0}\right) e \leq_{C} 0\right\}$. Let $\Psi \subseteq X$ such that for every $\bar{x} \in S_{0} \backslash \Psi$ there exists $x \in X$ such that $x \neq \bar{x}$ and $f(\bar{x}, x)+\varepsilon \alpha_{\mu} p_{\mu}(x-\bar{x}) e \leq_{C} 0$. Then $S_{0} \cap \Psi \neq \emptyset$.

Proof. By Theorem 2.1 there exists $z \in S_{0}$ such that satisfies the condition (ii) in Theorem 2.1. It is easy to see that $z \in S_{0} \cap \Psi$.

\section{Equivalences}

In this section, we show that Daneš' drop theorem (Theorem 1.3), two versions of vectorial form of Ekeland-type variational principle, vectorial Caristi-Kirk's fixed-point theorem and vectorial Takahashi's nonconvex minimization theorem are equivalent. In order to show that Theorems 1.3 and 2.1 are equivalent to each other we need the following definition which was introduced by Cheng et al. [34].

Definition 4.1. Two nonempty subsets $A$ and $B$ of the locally convex space $E$ are said to be strongly Minkowski separated if and only if there exist a continuous seminorm $p$ and $z \in E$ such that either

$$
\inf \{p(a+z): a \in A\}>\sup \{p(b+z): b \in B\}
$$

or

$$
\sup \{p(a+z): a \in A\}<\inf \{p(b+z): b \in B\}
$$

Theorem 4.2. Theorems 2.1 and 1.3 are equivalent to each other.

Proof. It is only enough to show that Theorem 2.5 implies Theorem 1.3. Since $0 \notin \mathrm{cl}(A-B)$, then there exist $\eta \in \Lambda$ and $\delta>0$ such that

$$
p_{\eta}(a-b) \geq \delta, \quad \forall a \in A, b \in B .
$$


Therefore, by Lemma 1 of [7] $A$ and $B$ are strongly Minkowski separated. Hence, there exist a continuous seminorm $p$ and $z \in E$ such that

$$
\inf \{p(a+z): a \in A\}>\sup \{p(b+z): b \in B\} .
$$

Without loss of generality we may assume that $z=0$ and put

$$
d_{1}:=\inf \{p(x)-p(y): x \in A, y \in b\} .
$$

Also, $B$ is bounded, thus $d_{2}:=\sup \left\{p(x-y): x, y \in D\left(x_{0}, B\right)\right\}$ is finite. Now, we apply Theorem 2.5 for the set $X=D\left(x_{0}, B\right) \cap A$, function $f(x, y):=e(p(y)-p(x)), \varepsilon=1$ and a positive number $\alpha$ which $\alpha \leq d_{1} / d_{2}$. It is easy to see that the assumptions (A1)-(A4) are satisfied. If $A$ or $B$ is locally complete, then $X$ is locally complete, so $\left\{x \in X: f\left(x_{0}, x\right) \leq_{C} 0\right\}$ is locally complete. Therefore, by Theorem 2.5 there exists a point $z \in X$ such that

$$
f(z, x)+\alpha p(x-z) e \mathbb{Z}_{C} 0, \quad \forall x \in X, x \neq z .
$$

Let $x \in D(z, B) \cap A$, then $x=t z+(1-t) b$, where $b \in B$ and $0 \leq t \leq 1$. Hence,

$$
\begin{aligned}
\xi_{e}(f(z, x)+\alpha p(x-z) e) & =p(t z+(1-t) b)-p(z)+\alpha p((1-t)(b-z)) \\
& \leq t p(z)+(1-t) p(b)+\alpha(1-t) p(b-z)-p(z) \\
& \leq t p(z)+(1-t) p(z)-d_{1}+\alpha d_{2}-p(z) \\
& \leq 0 .
\end{aligned}
$$

Therefore, $f(z, x)+\alpha p(x-z) e \in-C$ and so by (4.6), we conclude that $x=z$.

Theorem 4.3. Theorems 2.1, 2.5, 3.3, 3.5 and 3.7 are mutually equivalent.

Proof. (1) Theorem $2.5 \Leftrightarrow$ Theorem 2.1.

It is enough to show that Theorem $2.5 \Rightarrow$ Theorem 2.1. Choose

$$
S=\bigcap_{\lambda \in \Lambda}\left\{x \in X: \alpha_{\lambda} p_{\lambda}(x) \leq 1\right\} .
$$

Then $S \subset X$ is a bounded, closed absolutely convex set. If $p_{S}$ is the Minkowski functional of $S$, then

$$
p_{S}(x)=\sup _{\lambda \in \Lambda} \alpha_{\lambda} p_{\lambda}(x), \quad \forall x \in X
$$

Now, if we apply Theorem 2.5 for the set $S$, then we obtain Theorem 2.1.

(2) Theorem $3.3 \Leftrightarrow$ Theorem 2.1.

It is enough to show that Theorem $3.3 \Rightarrow$ Theorem 2.1. Define $T: X \rightarrow 2^{X}$ as follows:

$$
T x=\left\{y \in X: f(x, y)+\alpha_{\curlywedge} p_{\lambda}(y-x) e \leq_{C} 0, \forall \lambda \in \Lambda\right\} .
$$


Obviously, for any $x \in X, T x \neq \emptyset$. And for each $x \in X$ and $y \in T x$,

$$
f(x, y)+\alpha_{\lambda} p_{\lambda}(y-x) e \leq_{C} 0, \quad \forall \lambda \in \Lambda .
$$

By Theorem 3.3, there exists $z \in T x_{0}$ such that $T z=\{z\}$. Therefore, $x \notin T z$ for any $x \in X$, $x \neq z$. Thus, there exists $\mu \in \Lambda$, such that $f(z, x)+\alpha_{\mu} p_{\mu}(x-z) e \mathbb{L}_{C} 0$. Hence, the part (ii) in Theorem 2.1 holds.

(3) Theorem $3.5 \Leftrightarrow$ Theorem 2.1.

It is enough to show that Theorem $3.5 \Rightarrow$ Theorem 2.1. Without loss of generality we assume that $\varepsilon=1$. Let

$$
X_{0}=\left\{x \in X: f\left(x_{0}, x\right)+\alpha_{\curlywedge} p_{\lambda}\left(x-x_{0}\right) e \leq_{C} 0, \forall \lambda \in \Lambda\right\} .
$$

Since $x_{0} \in X_{0}$, then $X_{0}$ is nonempty. Also, for any $x \in X, f(x, \cdot)$ is $(e, C)$-locally lower semi continuous, thus $X_{0}$ is locally closed. Hence, if $\left\{x \in X: f\left(x_{0}, x\right) \leq_{C} 0\right\}$ is locally complete, then $\left\{x \in X_{0}: f\left(x_{0}, x\right) \leq_{C} 0\right\}$ is locally complete. Suppose that the part (ii) of Theorem 2.1 does not hold, that is, for all $x \in X_{0}$ there exists $w \neq x$ such that

$$
f(x, w)+\alpha_{\lambda} p_{\lambda}(w-x) e \leq_{C} 0, \quad \forall \lambda \in \Lambda .
$$

Therefore,

$$
\begin{aligned}
& f\left(x_{0}, w\right)+\alpha_{\lambda} p_{\lambda}\left(w-x_{0}\right) e \\
& \quad \leq_{C} f\left(x_{0}, w\right)+\alpha_{\lambda} p_{\lambda}(w-x) e+\alpha_{\lambda} p_{\lambda}\left(x-x_{0}\right) e \\
& \quad \leq_{C} f(x, w)+f\left(x_{0}, x\right)+\alpha_{\lambda} p_{\lambda}(w-x) e+\alpha_{\lambda} p_{\lambda}\left(x-x_{0}\right) e \\
& \quad \leq_{C} 0 .
\end{aligned}
$$

Hence, $w \in X_{0}$, so by Theorem 3.5, there exists $\bar{x} \in X_{0}$ such that $f\left(x_{0}, X\right) \cap\left(f\left(x_{0}, \bar{x}\right)-C\right)=$ $\left\{f\left(x_{0}, \bar{x}\right)\right\}$.

However, there exists $\bar{w} \in X_{0}$ such that $\bar{w} \neq \bar{x}$ which satisfies (4.13). Therefore, $f(\bar{x}, \bar{w}) \leq_{C} 0$ and so $f\left(x_{0}, \bar{w}\right) \leq_{C} f\left(x_{0}, \bar{x}\right)+f(\bar{x}, \bar{w}) \leq_{C} f\left(x_{0}, \bar{x}\right)$, which is a contradiction.

(4) Theorem $3.7 \Leftrightarrow$ Theorem 2.1.

It is enough to show that Theorem $3.7 \Rightarrow$ Theorem 2.1. Suppose that $T: X \rightarrow 2^{X}$ is defined as follows:

$$
T x=\left\{y \in X: f(x, y)+\varepsilon \alpha_{\curlywedge} p_{\lambda}(y-x) e \leq_{C} 0, \forall \lambda \in \Lambda\right\} .
$$

Choose $\Psi=\{x \in X: T(x)=\{x\}$. If $x \notin \Psi$, then there exists $y \in T(x)$ such that $y \neq x$. Therefore, assumption of Theorem 3.7 is satisfied. Hence, there exists $z \in S_{0} \cap \Psi$. From the definition of $\Psi$ the results (i) and (ii) of Theorem 2.1 are satisfied.

Remark 4.4. (a) By the same proof as that of Theorem 4.3, one can show that Theorems 2.5, 3.4, and 3.6 are equivalent to each other.

(b) By the same proof as that of Theorems 5.2 and 5.3 in [18] one can prove that Theorem 2.1 and the Phelp's lemma ([18, Theorem 5.1]) are equivalent. 


\section{Acknowledgment}

M. FAKHAR was partially supported by the Center of Excellence for Mathematics, University of Isfahan.

\section{References}

[1] I. Ekeland, "On the variational principle," Journal of Mathematical Analysis and Applications, vol. 47, pp. 324-353, 1974.

[2] J.-P. Aubin and I. Ekeland, Applied Nonlinear Analysis, Pure and Applied Mathematics (New York), John Wiley \& Sons, New York, NY, USA, 1984.

[3] G. Y. Chen and X. X. Huang, "A unified approach to the existing three types of variational principles for vector valued functions," Mathematical Methods of Operations Research, vol. 48, no. 3, pp. 349-357, 1998.

[4] G.-Y. Chen, X. Huang, and X. Yang, Vector Optimization. Set-Valued and Variational Analysis, vol. 541 of Lecture Notes in Economics and Mathematical Systems, Springer, Berlin, Germany, 2005.

[5] A. Göpfert, Chr. Tammer, and C. Zălinescu, "On the vectorial Ekeland's variational principle and minimal points in product spaces," Nonlinear Analysis: Theory, Methods \& Applications, vol. 39, no. 7, pp. 909-922, 2000.

[6] A. Göpfert, H. Riahi, Chr. Tammer, and C. Zălinescu, Variational Methods in Partially Ordered Spaces, vol. 17 of CMS Books in Mathematics, Springer, New York, NY, USA, 2003.

[7] A. H. Hamel, "Phelps' lemma, Daneš' drop theorem and Ekeland's principle in locally convex spaces," Proceedings of the American Mathematical Society, vol. 131, no. 10, pp. 3025-3038, 2003.

[8] G. Isac, "Nuclear cones in product spaces, Pareto efficiency and Ekeland-type variational principles in locally convex spaces," Optimization, vol. 53, no. 3, pp. 253-268, 2004.

[9] G. Isac and Chr. Tammer, "Nuclear and full nuclear cones in product spaces: pareto efficiency and an Ekeland type variational principle," Positivity, vol. 9, no. 3, pp. 511-539, 2005.

[10] L.-J. Lin and W.-S. Du, "Ekeland's variational principle, minimax theorems and existence of nonconvex equilibria in complete metric spaces," Journal of Mathematical Analysis and Applications, vol. 323, no. 1, pp. 360-370, 2006.

[11] C. Gutiérrez, B. Jiménez, and V. Novo, "A set-valued Ekeland's variational principle in vector optimization," SIAM Journal on Control and Optimization, vol. 47, no. 2, pp. 883-903, 2008.

[12] Q. H. Ansari, "Vectorial form of Ekeland-type variational principle with applications to vector equilibrium problems and fixed point theory," Journal of Mathematical Analysis and Applications, vol. 334, no. 1, pp. 561-575, 2007.

[13] Y. Araya, “Ekeland's variational principle and its equivalent theorems in vector optimization," Journal of Mathematical Analysis and Applications, vol. 346, no. 1, pp. 9-16, 2008.

[14] C. Finet and L. Quarta, "Vector-valued perturbed equilibrium problems," Journal of Mathematical Analysis and Applications, vol. 343, no. 1, pp. 531-545, 2008.

[15] C. Finet, L. Quarta, and C. Troestler, "Vector-valued variational principles," Nonlinear Analysis: Theory, Methods \& Applications, vol. 52, no. 1, pp. 197-218, 2003.

[16] W. Oettli and M. Théra, "Equivalents of Ekeland's principle," Bulletin of the Australian Mathematical Society, vol. 48, no. 3, pp. 385-392, 1993.

[17] J.-H. Qiu, "Local completeness and drop theorem," Journal of Mathematical Analysis and Applications, vol. 266, no. 2, pp. 288-297, 2002.

[18] J.-H. Qiu, "Local completeness, drop theorem and Ekeland's variational principle," Journal of Mathematical Analysis and Applications, vol. 311, no. 1, pp. 23-39, 2005.

[19] Z. Wu, "Equivalent formulations of Ekeland's variational principle," Nonlinear Analysis: Theory, Methods E Applications, vol. 55, no. 5, pp. 609-615, 2003.

[20] M. Bianchi, G. Kassay, and R. Pini, “Ekeland's principle for vector equilibrium problems," Nonlinear Analysis: Theory, Methods \& Applications, vol. 66, no. 7, pp. 1454-1464, 2007.

[21] G.-Y. Chen, X. Q. Yang, and H. Yu, "Vector Ekeland's variational principle in an F-type topological space," Mathematical Methods of Operations Research, vol. 67, no. 3, pp. 471-478, 2008.

[22] T. X. D. Ha, "Some variants of the Ekeland variational principle for a set-valued map," Journal of Optimization Theory and Applications, vol. 124, no. 1, pp. 187-206, 2005. 
[23] M. Bianchi, N. Hadjisavvas, and S. Schaible, "Vector equilibrium problems with generalized monotone bifunctions," Journal of Optimization Theory and Applications, vol. 92, no. 3, pp. 527-542, 1997.

[24] M. Fakhar and J. Zafarani, "Generalized vector equilibrium problems for pseudomonotone multivalued bifunctions," Journal of Optimization Theory and Applications, vol. 126, no. 1, pp. 109-124, 2005.

[25] J. Zhu, C. K. Zhong, and Y. J. Cho, “Generalized variational principle and vector optimization,” Journal of Optimization Theory and Applications, vol. 106, no. 1, pp. 201-217, 2000.

[26] S. Al-Homidan, Q. H. Ansari, and J.-C. Yao, "Some generalizations of Ekeland-type variational principle with applications to equilibrium problems and fixed point theory," Nonlinear Analysis: Theory, Methods E Applications, vol. 69, no. 1, pp. 126-139, 2008.

[27] L.-J. Lin and W.-S. Du, "On maximal element theorems, variants of Ekeland's variational principle and their applications," Nonlinear Analysis: Theory, Methods E Applications, vol. 68, no. 5, pp. 12461262, 2008.

[28] P. Pérez Carreras and J. Bonet, Barrelled Locally Convex Spaces, vol. 131 of North-Holland Mathematics Studies, North-Holland, Amsterdam, The Netherlands, 1987.

[29] H. Jarchow, Locally Convex Spaces, B. G. Teubner, Stuttgart, Germany, 1981.

[30] M. Fakhar and J. Zafarani, "Equilibrium problems in the quasimonotone case," Journal of Optimization Theory and Applications, vol. 126, no. 1, pp. 125-136, 2005.

[31] F. Giannessi, Ed., Vector Variational Inequalities and Vector Equilibria. Mathematical Theories, vol. 38 of Nonconvex Optimization and Its Applications, Kluwer Academic Publishers, Dordrecht, The Netherlands, 2000.

[32] W. Oettli and D. Schläger, "Existence of equilibria for monotone multivalued mappings," Mathematical Methods of Operations Research, vol. 48, no. 2, pp. 219-228, 1998.

[33] C. Gerstewitz, "Nichtkonvexe Dualität in der Vektoroptimierung," Wissenschaftliche Zeitschrift der Technischen Hochschule für Chemie "Carl Schorlemmer", Leuna-Merseburg, vol. 25, no. 3, pp. 357-364, 1983.

[34] L. X. Cheng, Y. Zhou, and F. Zhang, "Danes' drop theorem in locally convex spaces," Proceedings of the American Mathematical Society, vol. 124, no. 12, pp. 3699-3702, 1996. 